\title{
RICERCA
}

\section{GESTIRE A DISTANZA IL DIABETE IN REGIME DI LOCKDOWN: UN'INDAGINE SUGLI ESITI CLINICI E PSICO-EDUCAZIONALI}

\author{
Benedetta Mainetti ${ }^{1}$, Teresa Ilaria Ercolanese ${ }^{1}$, \\ Antonella Liverani ${ }^{1}$, Aldo Vallicelli ${ }^{2}$, Enrico Valletta ${ }^{1}$ \\ ${ }^{1}$ UO di Pediatria, Ospedale "G.B. Morgagni - L. Pierantoni", Forli, AUSL della Romagna \\ ${ }^{2}$ Associazione Diabete Romagna ONLUS
}

Indirizzo per corrispondenza: benedetta.mainetti@auslromagna.it

\section{REMOTE MANAGEMENT OF DIABETES \\ IN A LOCKDOWN REGIME: A SURVEY ON CLINICAL AND PSYCHOEDUCATIONAL OUTCOMES}

\author{
Key words \\ SARS-CoV-2, Lockdown, Type 1 diabetes, HbA1c, BMI, \\ Psychology
}

\begin{abstract}
During the lockdown for the SARS-CoV-2 epidemic in Italy, paediatric Diabetology Centres reorganized their activity and started to follow their patients at a distance. The risk of worsening disease control seemed realistic taking in account the interruption of scheduled visits, home confinement and the consequent reduction of physical activity. Our Service quickly made the telemedicine resources its own, continuing the clinical monitoring activity and the psycho-educational work of accompanying families from remote. The analysis of the HbAlc and BMI trend at the end of the lockdown suggests that no significant deterioration occurred in our 41 patients with type 1 diabetes (age < 18 years) during the three months of home quarantine. Some specific situations of adolescents with excess weight, behavioural problems and poor glycaemic control are discussed in more detail. The survey conducted on parents at the end of the quarantine seems to confirm initial fears: overall reduction of physical activity, prolonged use of social networks, less controlled nutrition especially in adolescents and evident repercussions on their emotional state. Some of these effects persist even after returning to normal social life. In summary, the work carried out by the Diabetology team, albeit at a distance, seems to have contained the unfavourable effects of lockdown on diabetes control and confirms the need for strong psychological support and proximity in similar situations of social isolation.
\end{abstract}

\section{RIASSUNTO}

Durante il lockdown, conseguente all'epidemia di SARSCoV-2 in Italia, i Centri di Diabetologia pediatrica hanno dovuto riorganizzarsi per continuare a seguire a distanza i propri pazienti. Il rischio di un peggioramento del controllo della malattia appariva realistico considerando l'interruzione delle visite programmate, il confinamento domiciliare e la conseguente riduzione dell'attività fisica. Anche il nostro Servizio ha rapidamente fatto proprie le risorse della telemedicina, proseguendo da remoto l'attività di monitoraggio clinico e il lavoro psico-educazionale di accompagnamento delle famiglie. L'analisi dell'andamento dell'emoglobina glicata e del Body Mass Index al termine del lockdown suggerisce che nessun significativo peggioramento si è verificato nei nostri 41 pazienti con diabete tipo 1 (età $<18$ anni) nel corso dei tre mesi di quarantena domiciliare. Alcune situazioni particolarmente difficili di adolescenti con eccesso ponderale, problematiche comportamentali e cattivo controllo glicemico vengono discussi più nel dettaglio. L'indagine condotta al termine del lockdown conferma quanto inizialmente temuto: complessiva riduzione dell'attività fisica, uso protratto dei social, alimentazione meno controllata soprattutto negli adolescenti e apprezzabili ripercussioni sul loro stato emotivo. Alcuni di questi effetti tendono a protrarsi anche dopo il ritorno alla normalità.

In sintesi, il lavoro che l'équipe diabetologica ha svolto, seppure a distanza, con le famiglie sembra avere contenuto gli effetti sfavorevoli sul controllo del diabete e conferma la necessità di un forte supporto psicologico e di vicinanza in analoghe situazioni di isolamento sociale.

\section{INTRODUZIONE}

Nella primavera del 2020, la pandemia di SARS-CoV-2 ha determinato la progressiva adozione di misure di contenimento del contagio che, in Italia, è culminata nel co- 
siddetto lockdown (LD), protrattosi per circa tre mesi. In quel periodo, le famiglie sono state confinate nelle loro case e anche le consuete attività sanitarie non urgenti risultavano sospese, inclusa l'attività del Servizio di Diabetologia pediatrica del nostro reparto. Al timore diffuso di un'infezione dagli esiti ancora largamente sconosciuti nella popolazione infantile - e segnatamente nei soggetti affetti da patologie croniche - si aggiungeva la preoccupazione per gli effetti negativi che la forzata permanenza in spazi limitati e l'interruzione dei regolari controlli clinici avrebbero avuto sul controllo del diabete nei bambini e negli adolescenti. L'équipe ha così dovuto rapidamente riorganizzarsi. I contatti telefonici, la trasmissione dei diari e l'impiego degli strumenti informatici per la raccolta dei dati clinici a distanza hanno costituito la nuova modalità attraverso la quale far sentire la propria vicinanza alle famiglie e avere un costante monitoraggio dello stato di salute dei bambini e dei ragazzi con diabete ${ }^{1}$. L'obiettivo è stato quello di dare continuità al lavoro sulla malattia senza trascurare gli aspetti psicologici ed emotivi messi a dura prova dalla contingenza emergenziale e di proseguire il percorso psico-educazionale svolto - singolarmente o in gruppo - in presenza.

La fine del LD e il progressivo, lento, ritorno alla "normalità" hanno reso possibile un momento di riflessione e di verifica su quanto svolto, con modalità per noi inusuali, nel trimestre precedente. Abbiamo, quindi, concentrato la nostra attenzione sulle modifiche intercorse in due fondamentali indicatori clinici - emoglobina glicata (HbAlc) e Body Mass Index (BMI) - e su quanto avesse influito il LD relativamente ad alcuni importanti ambiti di vita dei bambini e degli adolescenti con diabete.

\section{PAZIENTI E LE NUOVE STRATEGIE DI "VICINANZA"}

All'inizio del LD - che si è protratto dal 9 marzo al 31 maggio 2020 - erano 45 i bambini o adolescenti con diabete tipo 1 (DT1) seguiti presso la nostra Pediatria. Fino ad allora, l'équipe diabetologica (costituita da una pediatra, due psicologhe, un dietista e due infermiere dedicate all'ambulatorio) aveva svolto, in presenza, una regolare attività di monitoraggio clinico e di accompagnamento psico-educazionale volto a favorire l'adattamento e il controllo della malattia e ad assicurare un adeguato sostegno ai pazienti e alle loro famiglie.

Nei tre mesi del LD, sospesi gli abituali controlli ambulatoriali, sono state rapidamente adottate modalità di telemedicina mantenendo le scadenze degli appuntamenti già programmati. Tramite i dispositivi (glucometri, sensori glicemici, microinfusori) collegati ai rispettivi siti web, è stato possibile mantenere il controllo di ciascuno, scaricando i dati (andamento glicemico, fabbisogno insulinico) e contattando poi le famiglie telefonicamente. Nel corso dei colloqui venivano discussi gli aspetti relativi al controllo della glicemia, adeguando gli interventi alla nuova quotidianità (diversi orari, nuovo stile di vita, diverso tipo di alimentazione), ma si cercava anche di dare sostegno ai genitori rispetto alla percepita "fragilità" dei propri figli. Concluso il LD, nell'arco dei due mesi successivi, per tutti i pazienti sono stati programmati controlli in presenza con verifica del BMI e dell'HbAlc. Si è quindi provveduto a confrontare i risultati ottenuti prima (pre-LD) e dopo (post-LD) il LD, suddividendo i pazienti in tre fasce di età: prescolare e scolare $(<10$ anni), preadolescenza (1114 anni) e adolescenza (15-18 anni).

Anche dal punto di vista psico-educazionale, durante il LD sono state previste modalità di contatto a distanza che mantenessero inalterati i consueti tempi e orari in concomitanza con l'attività ambulatoriale della pediatra diabetologa. L'invio e la discussione dei materiali sono stati orientati a sostenere il benessere psicofisico del bambino e dell'adolescente (come affrontare la situazione in atto, l'incremento del tempo libero, la noia e il senso di spaesamento) e non esclusivamente alla sua condizione diabetica. Gli stimoli suggeriti ai genitori venivano diversificati in base alle tematiche proposte (cultura, spettacolo, tempo libero, approcci psicoeducativi) e alle fasce di età dei bambini e degli adolescenti a cui erano rivolti. L'attenzione al paziente era, allo stesso tempo, attenzione ai genitori - coinvolti e responsabilizzati verso il mantenimento di un corretto stile di vita - e al benessere psicofisico dell'intera famiglia. All'interno del gruppo, i messaggi inviati avevano un'intestazione comune - "... homemade" - con un richiamo alla condizione domestica ma anche alla produzione personale di opere e azioni che potessero essere di aiuto nell'affrontare la situazione del momento.

Terminato il LD, abbiamo ritenuto fosse importante raccogliere il punto di vista delle famiglie rispetto all'esperienza della quarantena, per valutarne l'impatto sulla gestione complessiva della malattia. Abbiamo, quindi, proposto ai genitori un questionario che mirava a indagare lo stile di vita dei nuclei familiari prima, durante e dopo il periodo della quarantena. Gli ambiti che abbiamo inteso approfondire sono stati quello dell'attività fisica e delle abitudini alimentari. Una sezione specifica è stata pensata per i genitori con figli adolescenti (> 11 anni) con l'intento di raccogliere informazioni sulle attività extrascolastiche svolte nei momenti liberi dallo studio e sullo stato emotivo osservato nei ragazzi. Infine, è stata richiesta una valutazione dell'intervento a distanza messo in atto dall'équipe diabetologica nei tre mesi di LD.

I dati, ove opportuno, sono stati analizzati utilizzando il Wilcoxon signed-rank test per dati appaiati, il test di Kruskal-Wallis per i confronti tra gruppi e il test esatto di Fisher. Il limite della significatività è stato posto per $\mathrm{p}<0,05$.

\section{ESITI CLINICI E ASPETTI PSICO-EDUCAZIONALI}

\section{Emoglobina glicata e Body Mass Index}

È stato possibile raccogliere i dati relativi ai livelli di HbAlc in 41 dei 45 pazienti seguiti dall'équipe diabetologica: 14 (6 maschi) erano tra 5 e 10 anni di età, 9 (4 maschi) tra 11 e 14 anni e 18 (8 maschi) tra 15 e 18 anni. 4 
pazienti sono stati esclusi dall'indagine poiché la loro transizione al Centro antidiabetico degli adulti, già in programma da diversi mesi, è avvenuta proprio in seguito al LD. La visita di controllo, pertanto, non è più stata effettuata nel nostro ambulatorio. Dei 41 pazienti, 29 (70,7\%) erano in MDI (Multiple Daily Injection, terapia multiniettiva) e $12(29,2 \%)$ erano in CSII (Continous Subcutaneous Insulin Infusion, microinfusore); di questi ultimi, 8 erano in SAP (Sensor Augmented Pump, microinfusore collegato a sensore). Per il monitoraggio della glicemia, 23 pazienti (56\%) utilizzavano il sistema FGM (Flash Glucose Monitoring, monitoraggio glicemico flash), 15 (36,5\%) il sistema CGM (Continous Glucose Monitoring, monitoraggio glicemico in continuo) e solo $3(7,3 \%) \mathrm{im}-$ piegavano il glucometro semplice con misurazione della glicemia capillare. Per i pazienti che utilizzavano FGM e CGM è stata utilizzata la telemedicina tramite i siti web: Freestyle Librelink, Carelink, Diasend e Clarity. Per i 3 pazienti che utilizzavano il glucometro semplice, il controllo a distanza è avvenuto con l'invio di foto del diario glicemico tramite WhatsApp.

I valori di $\mathrm{HbA} 1 \mathrm{c}$ sono risultati significativamente $(\mathrm{p}<$ $0,001)$ differenti nei tre gruppi sia nel pre-LD che nel post-LD, con valori più elevati nel sottogruppo di età 1114 anni. Nessuna variazione significativa tra pre-LD e post-LD è stata osservata sia nella popolazione totale dei pazienti $(\mathrm{p}=0,49)$ sia nei tre gruppi individuati per età $(<$ 10 anni, $\mathrm{p}=0,88 ; 11-14$ anni, $\mathrm{p}=0,55 ; 15-18$ anni, $\mathrm{p}=$ 0,53) (Tabella I). Il BMI, espresso come deviazione standard (DS) per sesso ed età (GROWTH4, Weboriented, SIEDP) non ha mostrato differenze significative tra i tre gruppi sia nel pre-LD $(\mathrm{p}=0,96)$ che nel post-LD ( $\mathrm{p}=$ $0,92)$ né apprezzabili modificazioni tra pre-LD e post-LD in nessuno dei gruppi singolarmente considerati $(<10$ anni, $\mathrm{p}=0,14 ; 11-14$ anni, $\mathrm{p}=0,68 ; 15-18$ anni, $\mathrm{p}=0,84$; totale, $\mathrm{p}=0,41)$.

\begin{tabular}{|l|c|c|c|c|c|}
\hline \multicolumn{5}{|c|}{$\begin{array}{c}\text { CONFRONTO TRA I VALORI DI EMOGLOBINA GLICATA } \\
\text { E DI BODY MASS INDEX PRIMA E DOPO IL LOCKDOWN }\end{array}$} \\
\hline & & \multicolumn{2}{|c|}{$\begin{array}{c}\text { Emoglobina glicata [\%] } \\
\text { mediana (min; max) }\end{array}$} & \multicolumn{2}{|c|}{$\begin{array}{c}\text { Body Mass Index [DS] } \\
\text { mediana (min; max) }\end{array}$} \\
\cline { 4 - 7 } & n & Pre-LD* & Post-LD* & Pre-LD & Post-LD \\
\hline$<\mathbf{1 0}$ anni & 14 & $7,1(6 ; 8,6)$ & $7(6,1 ; 9)$ & $0,1(-1,36 ; 1,56)$ & $-0,15(-2,55 ; 1,37)$ \\
\hline $\mathbf{1 1 - 1 4}$ anni & 9 & $7,8(6,4 ; 9,5)$ & $8,1(6,6 ; 9,6)$ & $0,45(-0,53 ; 1,07)$ & $0,47(-0,45 ; 1,6)$ \\
\hline $\mathbf{1 5 - 1 8}$ anni & 18 & $7,3(6,4 ; 9,3)$ & $7,45(6,6 ; 10)$ & $0,08(-1,89 ; 2,36)$ & $0,10(-1,48 ; 2,64)$ \\
\hline Totale & 41 & $7,4(6 ; 9,5)$ & $7,4(6,1 ; 10)$ & $0,12(-1,89 ; 2,36)$ & $0,08(-2,55 ; 2,64)$ \\
\hline
\end{tabular}

Tabella I. *Confronto tra gruppi: $p<0,001$. Restanti confronti tra gruppi o pre-LD vs post-LD: $p=n s$.

\section{Indagine psico-educazionale}

È stato possibile condurre l'indagine tramite questionario in 31 famiglie: in 13 era presente un bambino/a in età prescolare o scolare $(<10$ anni) e in 18 un ragazzo/a preadolescente o adolescente (11-18 anni). Tutte le famiglie hanno risposto ai quesiti riguardanti gli ambiti dell'attività fisica, dell'alimentazione e della valutazione dell'intervento a distanza; 18 famiglie nelle quali era presente un ragazzo/a in età pre-adolescenziale o adolescenziale hanno risposto anche alla sezione specificamente dedicata.

\section{Attività fisica}

Dall'indagine è emerso che il $74 \%$ dei bambini/ragazzi era solito praticare regolarmente un'attività sportiva in epoca pre-LD e il $95 \%$ di loro lo faceva con una frequenza di almeno 1-3 volte/settimana. Il 90\% dei genitori riferisce che il proprio figlio/a svolgeva una significativa attività fisica libera (almeno 40 minuti, 1-3 volte/settimana) durante il pre-LD, percentuale che è scesa al 39\% durante il LD per poi risalire al $68 \%$ nel post-LD (post-LD vs preLD: $p=0,06$; pre-LD e post-LD vs LD: $p<0,01)$. Il 61\% dei genitori ritiene che il periodo di quarantena abbia modificato - in senso negativo - l'atteggiamento del figlio nei confronti dell'attività fisica anche nel post-LD.

\section{Abitudini alimentari}

Il $52 \%$ e il $68 \%$ dei genitori ritengono che l'alimentazione dei propri figli prima della quarantena fosse, rispettivamente, ben bilanciata e complessivamente regolare negli orari. Le risposte positive provengono, in prevalenza, dalle famiglie con figli adolescenti. Solo un terzo $(32 \%)$ dei genitori riporta un incremento dell'apporto alimentare durante il LD, prevalentemente (91\%) sotto forma di spuntini e merende fuori pasto. La maggioranza $(81 \%)$ delle famiglie intervistate riporta un consumo invariato di frutta e verdura nel corso del LD avendo l'impressione nel $71 \%$ dei casi che il periodo di quarantena non abbia determinato sostanziali variazioni delle abitudini alimentari.

\section{Adolescenza}

Secondo il 78\% dei genitori, i propri figli avrebbero incrementato, nel periodo del LD l'uso del computer e dello 
smartphone: in metà dei casi si trattava di oltre 4 ore/die e nel $17 \%$ oltre 6 ore/die. L'utilizzo degli strumenti informatici aveva diversi obiettivi che sono sintetizzati nella Figura 1. In circa metà dei casi, l'abitudine a trascorrere una maggiore quantità di tempo sugli strumenti informatici sembra protrarsi anche dopo il termine del LD.

Nel 39\% dei casi, le famiglie hanno l'impressione che nel corso della quarantena, il tono dell'umore dei ragazzi/e abbia subito un cambiamento, spesso di senso negativo ma talora anche in direzione opposta (Figura 2).

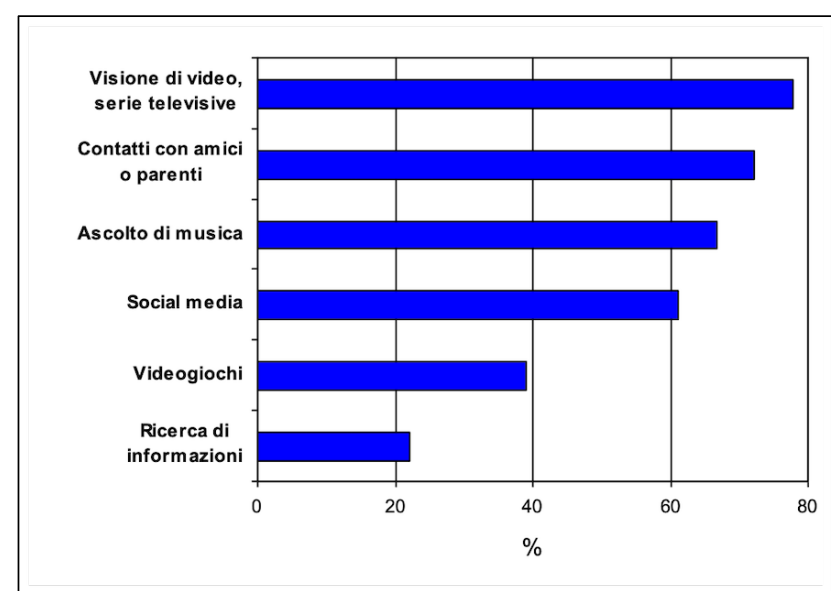

Figura 1

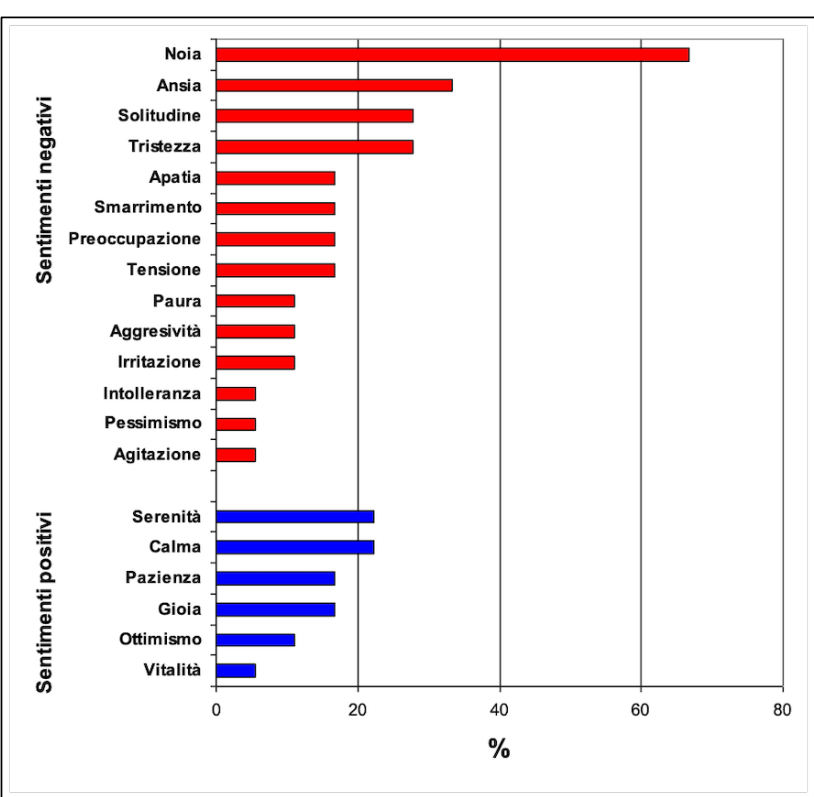

Figura 2

\section{Valutazione dell'intervento}

Il mantenimento, seppure a distanza, dei regolari rapporti da parte dell'équipe diabetologica con le famiglie e i ragazzi è stata valutata positivamente nel $66 \%$ dei casi, soprattutto dai genitori degli adolescenti. Lo scambio di esperienze nel gruppo dei genitori, la promozione dell'attività fisica e l'affiancamento nella gestione quotidiana del diabete sono risultati essere gli aspetti più apprezzati.

\section{DISCUSSIONE}

Analogamente a quanto osservato in altri Centri di Diabetologia pediatrica, il LD non sembra avere influito significativamente sul controllo glicemico, nonostante i cambiamenti nello stile di vita e l'impossibilità a svolgere i consueti controlli e a confrontarsi in presenza con l'équipe diabetologica. Dati preliminari di letteratura segnalano addirittura la possibilità di un migliore controllo glicemico in bambini e adulti con DT1 nelle prime settimane del $\mathrm{LD}^{2-4}$. I motivi possono essere diversi: la diminuzione degli impegni quotidiani ha consentito agli adulti una maggiore attenzione alla cura della propria malattia, mentre $\mathrm{i}$ genitori dei bambini più piccoli hanno avuto più tempo da dedicare a loro e alla gestione del diabete, curando maggiormente anche la preparazione dei pasti. Nella fase successiva al LD, alcuni studi hanno rilevato nei bambini un miglioramento del controllo glicemico rispetto a prima e negli adolescenti un buon controllo glicemico non solo durante il LD ma anche nelle settimane successive, ritenendo l'assistenza prestata tramite telemedicina un aspetto determinante di questo risultato ${ }^{5,6}$.

Anche nel nostro gruppo di famiglie, i genitori si sono dimostrati più disponibili a seguire i piccoli o a concordare con i più grandi la terapia. Mai come in questo tempo $\mathrm{i}$ social sono stati utili per rafforzare la rete di comunicazione tra l'équipe diabetologica e le famiglie. Le risorse della telemedicina, già da anni utilizzata negli ambulatori di Diabetologia, hanno consentito di sostituire efficacemente la consulenza medica in presenza. La visualizzazione dei dati glicemici sui siti web connessi ai glucometri, ai sensori glicemici e ai microinfusori, ha permesso il confronto costante e un maggiore coinvolgimento dei genitori nel trovare soluzioni o aggiustamenti per la terapia insulinica $^{7,8}$. Esperienze internazionali confermano che l'adozione di analoghe modalità di visita telematica e di promozione di percorsi educazionali sui social hanno ottenuto risultati complessivamente soddisfacenti, riportando un apprezzabile coinvolgimento e grado di soddisfazione da parte delle famiglie ${ }^{9}$.

Nella nostra esperienza, tutti i bambini di età inferiore ai 10 anni hanno mantenuto un buon controllo glicemico durante il LD, con HbA1c post-LD sovrapponibili a quelle del periodo precedente, senza apprezzabili variazioni del BMI. Verosimilmente, i bambini di questa fascia di età si sono giovati di una più costante presenza dei genitori, con un ritmo di vita e orari dei pasti molto regolari, hanno mantenuto l'impegno scolastico al mattino e nelle ore pomeridiane hanno praticato un'attività fisica regolare, spesso in compagnia dei genitori. Rispetto ai mesi precedenti, hanno vissuto la quotidianità con ritmi meno incalzanti e stressanti venendo a mancare, per molti di loro, l'orario scolastico a tempo pieno, la mensa, gli impegni pomeridiani e l'avvicendarsi di diverse figure di riferimento. Naturalmente, non per tutti è stato così: il caso descritto nel Box 1 mette in evidenza come l'emergenza Covid-19 abbia peggiorato la condizione di fragilità di alcuni nuclei familiari già duramente provati in epoca pre-LD ${ }^{10}$. 


\section{BoX 1 - OMAR: COME IL LOCKDOWN PUÒ AMPLIFICARE LE DISUGUAGLIANZE}

Omar ha 10 anni e due anni prima ha avuto l'esordio di diabete in Marocco. A 9 anni, con la mamma e il fratellino, parte alla volta dell'Italia per ricongiungersi con il padre. Il viaggio non è semplice perché, già in Spagna, insorgono problemi per la regolarizzazione dei documenti. Arrivato in Italia, la convivenza con il padre dura solo pochi mesi e, tramite conoscenze, Omar con mamma e fratellino arrivano nella nostra città. In attesa di regolarizzare la loro posizione e potere garantire l'assistenza sanitaria a Omar, i Servizi Sociali riescono a organizzare vitto e alloggio presso la Caritas locale. Nel dicembre 2019, il valore della $\mathrm{HbA} 1 \mathrm{c}$ è $8,6 \%$ e, finalmente, riusciamo ad avviare l'adeguata assistenza per il diabete.

Pochi mesi ed è emergenza Covid-19, la famiglia resta confinata in un piccolo spazio abitativo e il necessario è ancora fornito dalla Caritas. Omar parla l'italiano, la mamma lo comprende, e riusciamo ad avere contatti telefonici durante il LD. Mancano del tutto gli strumenti per una visita telematica, ma è stato comunque possibile un monitoraggio attraverso l'invio di foto del diario glicemico tramite WhatsApp. $\mathrm{Ci}$ accontentiamo che venga proseguita la cura essenziale per il diabete: controlli glicemici regolari, insulina glargine al bed-time e insulina lispro prima dei pasti cercando, con difficoltà, di adattare la dose all'apporto di carboidrati. Alla visita, superato il LD, il controllo glicemico risulta purtroppo peggiorato con $\mathrm{HbAlc}$ pari a $9 \%$.

Il gruppo dei preadolescenti (11-14 anni), pur essendo quello più problematico dal punto di vista del controllo glicemico, ha mostrato una sostanziale stabilità dell'HbA1c e del BMI. Questa fascia di età ha sicuramente risentito sia della mancanza dell'attività sportiva, praticata generalmente a livello pre-agonistico, sia di un maggior tempo trascorso sui social e di un'alimentazione meno regolata e con un maggiore consumo di alimenti fuori pasto. Ancora più critico è stato il comportamento di un piccolo numero di ragazze tredicenni che avevano una HbAlc già molto alta prima del LD (HbA1c 9-9,3\%). Si tratta di preadolescenti che attraversano un periodo di rifiuto della malattia, con scarsa compliance alla terapia e iniziali atteggiamenti di tipo trasgressivo. Di queste, una è anche risultata positiva al Covid-19 (Box 2).

Nel gruppo degli adolescenti (14-18 anni) il comportamento complessivo è stato simile a quello osservato nei preadolescenti. Alcune ragazze, di 16-17 anni di età, già in condizione di grave obesità e con cattivo controllo glicemico, hanno mantenuto durante e dopo il LD il medesimo BMI con un pessimo controllo delle glicemie (HbAlc 9,5-10\%). Per queste ragazze, il LD ha determinato un ulteriore fattore di rischio per il loro stile di vita:

\section{Box 2 - LUCIA: IL DIABETE, IL COVID-19, LA DEPRESSIONE}

Lucia ha 14 anni, frequenta la terza media, ha il diabete dall'età di 5 anni. Con l'ingresso nell'adolescenza ha presentato un sensibile peggioramento del controllo glicemico al quale hanno contribuito diversi fattori: disagio nel far conoscere la propria malattia, desiderio di uniformarsi al gruppo dei coetanei, omissione dei regolari controlli glicemici, conflitto con i genitori, iniziali comportamenti trasgressivi. In preLD il valore di HbAlc è $9 \%$. Da alcuni mesi porta un sensore glicemico real-time dotato di allarmi. Poco dopo l'inizio del LD, uno dei genitori risulta positivo per SARS-CoV-2 e anche Lucia si contagia. La madre ce lo comunica con grande preoccupazione e noi avviamo uno stretto monitoraggio clinico a distanza. Lucia rimane scarsamente sintomatica. Attraverso il sito web, monitoriamo quotidianamente il profilo glicemico e ogni giorno ci sentiamo telefonicamente con la madre. Con Lucia abbiamo contatti più frequenti su WhatsApp. L'andamento glicemico è analogo a quello dei mesi precedenti con persistenza dei picchi glicemici postprandiali, pur con un iniziale miglioramento del profilo notturno. Quando dopo tre settimane il tampone di Lucia si negativizza, siamo più tranquilli, allentiamo i controlli e sospendiamo il monitoraggio quotidiano a distanza. Al controllo post-LD, il controllo glicemico appare tuttavia peggiorato (HbAlc 9,5\%) e ci rendiamo conto che, superato il rischio del Covid-19, è subentrato un importante stato depressivo. Il protrarsi del LD, la necessità di proseguire l'impegno scolastico con l'incognita dell'esame di terza media, la maggior consapevolezza del rischio affrontato da tutta la famiglia hanno contribuito a far trascurare ulteriormente la cura del diabete. Si è reso pertanto necessario avviare un percorso di sostegno psicologico.

assenza di attività fisica, alimentazione del tutto sregolata, eccessivo tempo trascorso sui social. A tutto ciò si è anche aggiunta l'ansia, condivisa da tutti i coetanei, per l'incognita riguardo gli esiti finali dell'anno scolastico. Nei fatti, è stato praticamente impossibile aiutarle poiché negavano il loro disagio e non erano disponibili a chiedere aiuto. Spesso hanno evitato di indossare il sensore glicemico rendendo così impossibile visualizzare l'andamento giornaliero. I genitori si sono rivelati poco consapevoli di queste criticità e i colloqui telefonici non sono stati di molta utilità. Solo al successivo controllo in ambulatorio è stato possibile condividere la gravità della situazione. Sempre nel gruppo degli adolescenti, ha creato grande preoccupazione un ragazzo di 16 anni per il quale si è reso necessario, prima della fine del LD, un ricovero per la progressiva insorgenza di una condotta di tipo hikikomori (Box 3). 


\section{Box 3 - ANDREA: DIABETE E HIKIKOMORI}

Andrea è un ragazzo di 16 anni, frequenta la seconda superiore, ha il diabete dall'età di 12 anni. Già prima del LD aveva iniziato a manifestare un profondo disagio personale: rifiuto della malattia, rapporto conflittuale con i genitori, problemi di socializzazione, peggioramento del rendimento scolastico, tendenza all'isolamento sociale con prolungata frequentazione del computer. L'HbAlc è 9,2\%. Rifiuta qualsiasi dispositivo tecnologico per la cura del diabete e non è disponibile ad avviare alcun percorso di sostegno psicologico. Solo i genitori hanno incontri regolari con la psicologa. Durante il LD, la mamma ci trasmette la sua preoccupazione per l'aggravarsi dell'isolamento sociale di Andrea, condizione che lei stessa, usando la recente terminologia, definisce hikikomori. Andrea trascorre la maggior parte della notte sui social, al mattino non riesce a svegliarsi e non segue le lezioni scolastiche on-line, ha sovvertito completamente l'orario dei pasti, non cura più l'igiene personale, trascura completamente i controlli glicemici. I genitori si preoccupano loro di effettuare i controlli - riferiscono ricorrenti picchi di iper/ipoglicemie. Riteniamo necessario ricoverare Andrea e cerchiamo, per lui, l'appoggio della Neuropsichiatria Infantile. Nonostante l'atteggiamento ostile del ragazzo, lo convinciamo a portare un sensore glicemico real-time con allarmi, che posizioniamo alla radice della coscia, sotto i boxer, nel punto meno visibile. Questo dispositivo, collegato ai cellulari dei genitori, si rivelerà molto utile per consentire finalmente un monitoraggio più affidabile. Andrea ha poi rifiutato di proseguire il percorso con il neuropsichiatra. Attualmente il profilo glicemico è in miglioramento con $\mathrm{HbAlc} 8,5 \%$.

I dati emersi dal questionario sottoposto alle famiglie confermano che la maggior parte dei nostri bambini e ragazzi, prima della quarantena, praticava un'attività motoria regolare. In linea con quanto riportato in letteratura, la nostra indagine segnala la difficoltà nel mantenere lo stesso grado di attività durante il $\mathrm{LD}$, determinando un atteggiamento di relativa "pigrizia" che ha prolungato i propri effetti anche nel periodo successivo ${ }^{11}$. Ridare continuità e mantenere alta la motivazione all'impegno sportivo non sembra facile, soprattutto dopo una pausa forzata come quella della quarantena. In una prospettiva futura di situazioni analoghe, sarà della massima importanza incoraggiare le famiglie alla prosecuzione di una regolare pratica motoria anche all'interno delle proprie abitazioni, valorizzandone gli aspetti positivi ${ }^{12}$. L'esercizio fisico regolare contribuisce a migliorare l'andamento glicemico e il benessere psicologico, riduce l'ansia, lo stress e agisce positivamente sull'umore e sulla qualità del sonno ${ }^{13}$.

Per certi versi, sorprende la convinzione riportata dalla maggior parte dei genitori che i propri figli adolescenti avessero, prima della quarantena, un'alimentazione regolare nei tempi e bilanciata nei nutrienti. Questo dato, appare un po' in contrasto con gli indici clinici di riferimento e con le nostre osservazioni nelle attività psicoeducative con i ragazzi, spesso in evidente difficoltà nell'aderire a un regime alimentare equilibrato. Riteniamo che questa lettura ottimistica da parte dei genitori possa originare da una parziale consapevolezza dei comportamenti reali dei propri figli adolescenti, spesso protesi verso le prime forme di autonomia e di trasgressione delle regole, incluse quelle alimentari.

Come già segnalato, nel corso del LD abbiamo osservato un incremento dell'impiego di computer e smartphone in età adolescenziale ${ }^{14}$. Tuttavia, non dobbiamo ignorare che il loro utilizzo ha avuto come principale finalità quella di mantenere le relazioni e favorire il contatto sociale, sfruttando il lato positivo dello strumento tecnologico in momenti di distanza fisica forzata. Consideriamo che l'adolescente, durante il LD, sia stato privato proprio di quella dimensione sociale che in genere lo spinge, in termini evolutivi, fuori dalla famiglia e verso la maturità adulta. Ora, i genitori percepiscono il rischio che questa nuova consuetudine di relazioni a distanza si mantenga nel tempo; rispetto a questo sarà importante mettere in atto strategie che possano aiutare i ragazzi a riconnettersi all'altro anche su un piano personale e di presenza.

Per quel che riguarda lo stato emotivo dei figli adolescenti durante il LD, il sentimento maggiormente osservato dai genitori è quello della noia, probabilmente espressione di un disagio percepito dai ragazzi rispetto alla situazione contingente. La noia adolescenziale, espressa nei momenti di sospensione dell'attività scolastica, contraddistingue questa fase evolutiva e spesso assume $\mathrm{i}$ tratti di un tempo vuoto e poco produttivo che spinge i ragazzi a cercare in altri canali vie di fuga alternative per l'impiego del tempo stesso $^{15}$. Le condotte di sregolatezza alimentare con un aumento di spuntini e merende fuori pasto potrebbero collocarsi in questa dinamica. La maggioranza dei genitori - soprattutto quelli con figli adolescenti - ha dato un giudizio positivo sulle modalità di mantenimento dei contatti a distanza con le famiglie. Questi genitori rappresentano, probabilmente, un sottogruppo che ha avvertito maggiormente la necessità di avere un appoggio, una condivisione da parte dell'équipe e anche un aiuto nella comprensione delle complesse dinamiche che si realizzano nel rapporto quotidiano con i figli adolescenti.

\section{CONCLUSIONI}

Ripercorrere la nostra recente esperienza ci ha consentito di riflettere sugli elementi critici che hanno caratterizzato il periodo di isolamento domiciliare nei nostri bambini e ragazzi con diabete ma, allo stesso tempo, di apprezzarne gli aspetti di resilienza complessiva che non sono mancati. L'abitudine al controllo della propria malattia e il lavoro psico-educazionale da sempre condotto in tempi di normalità hanno probabilmente contribuito a limitare i danni, in 
un periodo di isolamento potenzialmente pericoloso. L'impiego delle risorse della telemedicina, già sperimentata e ancor più rafforzata sul versante clinico, ha consentito di mantenere una vicinanza anche psicologica con le famiglie, affiancandole dal punto di vista educativo e guidandole nei momenti di maggiore difficoltà. Riteniamo che molto di quanto è stato sperimentato in questa contingenza emergenziale potrà esserci di aiuto, non solo in occasione di future situazioni analoghe, ma anche nell'abituale pratica assistenziale in tempi di normalità.

\section{Riconoscimenti}

L'impegno professionale della dottoressa Teresa Ilaria Ercolanese è sostenuto dall'Associazione Diabete Romagna ONLUS.

\section{BIBLIOGRAFIA}

[1] Tornese G, Scaramuzza A, Schiaffini R. Telemedicina ai tempi del coronavirus. Medico e Bambino 2020;39(3):142-3.

[2] Iughetti L, Trevisani V , Cattini U, et al. Covid-19 and type 1 diabetes: concerns and challenges. Acta Biomed 2020;91(3):e2020033. DOI: 10.23750/ abm.v91i3.10366.

[3] Bonora BM, Boscari F, Avogaro A, Bruttomesso D, Fadini GP. Glycaemic control among people with type 1 diabetes during lockdown for the SARS-CoV-2 outbreak in Italy. Diabetes Ther 2020;11(6):1369-79. DOI: 10.1007/s13300-02000829-7.

[4] Schiaffini R, Barbetti F, Rapini N, et al. School and preschool children with type 1 diabetes during COVID-19 quarantine: the synergic effect of parental control and technology. Diabetes Res Clin Pract 2020;166:108302. DOI: 10.1016/j.diabres. 2020.108302 .

[5] Di Dalmazi G, Maltoni G, Bongiorno C, et al. Comparison of the effects of lockdown due to Covid-19 on glucose patterns among children, adolescents, and adults with type 1 diabetes: CGM study. BMJ Open Diabetes Res Care 2020;8: e001664. DOI: 10.1136/bmjdrc-2020-001664.
[6] Ceconi V, Barbi E, Tornese G. Glycemic control in type 1 diabetes mellitus and Covid-19 lockdown: what comes after a "quarantine"? J Diabetes 2020;12(12):946-8. DOI: 10.1111/1753-0407.13110.

[7] McDonnel ME. Telemedicine in complex diabetes management. Curr Diab Rep 2018;18(7):42. DOI: 10.1007/s11892-018-1015-3.

[8] Clary L, Wang C, Byrne ME, Monaghan M. Covid-19 pandemic-related practices and policies affecting the continuity of behavioral health care among children with diabetes. Transl Behav Med 2020;10(4):819-26. DOI: 10.1093/tbm/ibaa072.

[9] Ziegler R. Challenges in the care of children and youth with diabetes in times of the corona pandemic: personal view of the situation in a German clinic. J Diabetes Sci Technol 2020;14(4):811-2. DOI: $10.1177 / 1932296820930281$.

[10] Brener A, Mazor-Aronovitch K, Rachmiel M, et al. Lessons learned from the continuous glucose monitoring metrics in pediatric patients with type 1 diabetes under Covid-19 lockdown. Acta Diabetol 2020; 57:1511-7. DOI: 10.1007/s00592-020-01596-4.

[11] Tortella P, Schembri R, Fumagalli G. Covid-19 e gli effetti dell'isolamento sulla sedentarietà e sull'attività fisica dei bambini: uno studio italiano. Formazione \& Insegnamento 2020;18(3):101-10.

[12] Tornese G, Ceconi V, Monasta L, Carletti C, Faleschini E, Barbi E. Glycemic control in type 1 diabetes mellitus during Covid-19 quarantine and the role of in-home physical activity. Diabetes Technol Ther 2020;22(6):462-7. DOI: 10.1089/dia. 2020.0169.

[13] Peluso MA, Guerra de Andrade LHS. Physical activity and mental health: the association between exercise and mood. Clinics (Sao Paulo) 2005;60(1):6170. DOI: 10.1590/s1807-5932200 5000100012.

[14] Pietrobelli A, Pecoraro L, Ferruzzi A, et al. Effects of Covid-19 lockdown on lifestyle behaviors in children with obesity living in Verona, Italy: a longitudinal study. Obesity 2020;28(8):1382-5. DOI: 10.1002/oby.22861.

[15] Diani C. La noia, questa sconosciuta: indagine su significati, emozioni e comportamenti associati a un costrutto tanto diffuso quanto confuso. Psicoterapeuti in Formazione 2018;21:46-66. 